\title{
Natural Media
}

\author{
Carl H. Smith* \\ LTRC, Ravensbourne \\ London, UK \\ c.smith@rave.ac.uk \\ Jazz Rasool \\ LTRC, Ravensbourne \\ London, UK \\ j.rasool@rave.ac.uk
}

\author{
Lena Korkovelou \\ LTRC, Ravensbourne \\ London, UK \\ l.korkovelou@rave.ac.uk
}

\author{
Nick Lambert \\ Ravensbourne \\ London, UK \\ n.lambert@rave.ac.uk
}

\author{
Robert Pepperell \\ FOVOLAB \\ Cardiff Metropolitan University \\ Cardiff, Wales, UK \\ rpepperell@cardiffmet.ac.uk
}

\author{
Tony Langford \\ FOVOLAB \\ Cardiff Metropolitan University \\ Cardiff, Wales, UK \\ tolangford@cardiffmet.ac.uk
}

\author{
Alistair Burleigh \\ FOVOLAB \\ Cardiff Metropolitan University \\ Cardiff, Wales, UK \\ aburleigh@cardiffmet.ac.uk
}

\begin{abstract}
Natural perception usually leads sensations to be able to be registered with awareness, but not necessarily be processed. This is typically a state developed with mindfulness practices. Unlike this natural registration-only perception, most current immersive technologies universally process information. Natural perception can stimulate subconscious registration without higher functioning processing. However, camera and other imaging-based technologies, especially digital devices, almost always initiate processing, reducing the possibility of subconscious registration typically engaged with during dreaming and other creative states of mind. Forms of media that adopt natural perception properties and behaviours can be considered to be natural media.
\end{abstract}

Perceptual technology. Redesigning media. New forms of experience. Wearable experience. Peripheral vision.

\section{WHAT IS NATURAL MEDIA?}

Clay Shirky's (2010) powerful metaphor describes media as the connective tissue of society. Media have been the connective tissue for civilisations through the ages, and have shaped the way cultures - particularly the media-dominated modern western culture -perceive and experience time and space. The evolution of media can be seen as a long journey - but as we experience the fourth wave of the digital era the journey has reached an unprecedented stage. From here, it is very difficult to predict the extent of the transformations that are to follow. More importantly, it is hard to fathom the consequences these will have on human beings not only as far as cognition goes but on human biology itself.

Media are no longer mere information carriers. Contemporary media literally have the power to create and reshape reality. Their very names demonstrate this quality openly -we talk of immersion, and of augmented and virtual realities. Different media environments (contexts) have always influenced our senses. Now, both our senses and our reality, our very place within space and time and our perception of these are radically reshaped by different media, the digital revolution in particular.

Marshal McLuhan (McLuhan 1967) analysed the interplay between media, the human senses, and reality, defining the two spaces created by the impact of different media on the senses as the "acoustic" and the "visual" space. The preliterate media tribal man used (the environment of the spoken word) created an acoustic space, in which humans acted naturally, seeing the world holistically and syncretically. From these natural media, we reached, via the literate media (scribal and print) and their emphasis on the visual, the age of the rampant rise of the digital. Print media has acted as the catalyst, marking a shift from the tribal/collective towards a more rational, analytical and individualistic context. The digital era has taken an even more radical step away from the collectivism and syncretism of the tribal, sensoriumdominated man. Digital media have brought about a world where everything is increasingly about the consumption/rapid processing and equally rapid dismissal/forgetting of content by digital representations of selves that gradually replace the physical ones. 
Unlike digital media, natural media emphasised registering (and the subliminal, subconscious influences this entails) rather than mechanised processing. The senses, including the peripheral senses, were crucial in the earlier stages of civilisation dominated by natural media. Intuition, imagination, instinct and emotion were emphasised in earlier cultures. As McLuhan (1967) wrote, until writing was invented man lived in a boundless, directionless space. Thanks to the written word, we entered the culture of linear perception of time, as language and the latter are inter-related. The written word shaped a visual space where all things are linear, organised by means of text. This emphasis on the visual has perhaps created a different kind of Plato's cave, where the chains of the senses are replaced by those of representation. More than ever before, our media put emphasis on a rational, even mechanistic and automated perception of reality. It is not so much the content of the new media (what is written/said) that transforms our brains, but the context (the actual interfaces and structures of the new media). For instance, on Facebook we learn to react with a limited, preselected range of emoticons, while the rapid processing content on "walls" (it is processed, but barely registered) arguably leads to ADHD and an increased feeling of distance and alienation.

As Harold Innis (Innis 1950) established, different media have certain time-space biases through which they shape social environments. Media which emphasise time are durable, while media which emphasise space are lighter and less durable (papyrus, paper). Media that emphasise time favour decentralisation, while those emphasising space favour systems of government that are less hierarchical in character.

While the senses concern space, mostly, (so do the media that emphasise them) representations function within time. Therefore, "as any time is "now" for the biological being, (who has a notion of time projections of the past and future, but represented only in the "now" by means of the arts, imagination, memory and grammar) any space is "here" for the digital being. Time must be manageable for the digital being in the same way space is for the physical. Humankind has already developed the capacity to make time more elastic (art, medicine, cosmetic surgery, etc.) but the real notion of manageable time has come with digital media, which can slow down, stop, repeat and reverse time. We are witnessing the implosion of everywhere into "here" and the explosion of the temporal "now" into several time dimensions. Time (attention span) is already the measure of value in the digital realm.
"The currency of future digital economics will be
clearly related to time-spending."
(Miroshnichenko 2016).

The media of the future are both light (occupy virtually no space, weigh nothing, much like paper) and durable, as they emphasise time. At the same time:

\begin{abstract}
"The network society shapes not horizontal but cloud-like structures of authority, with heavy centres and dispersed peripheries. This society is populated by humans (or rather their representations) and algorithms, many of which seek to act like humans." (Miroshnichenko 2016).
\end{abstract}

It is a hybrid society in more ways than one. In this digital reality, distance, directions, duration and timespan turn from physical characteristics to social ones. 'Angelism' (McLuhan 1967) and narcissism are the dominant emerging traits of this new reality: Electronic man has no physical body, as McLuhan put it, but is obsessed with the digital body of his representation instead. The digital environment allows him the sense of flight and an escape from physical, given reality. But the sense of flight is also countered by a new sense of gravity, which is social and psychological rather than physical: The absence of validation on the digital plane, of "likes" and approval, is experienced as a heavy sense of alienation and distance, much like one used to feel alone in a crowd. We already develop new senses and need - and lack - new terms to describe them. The new properties of the new environment reshape the properties of the new human beings.

Reversal of this situation is impossible, but the course could still be re-centred and a new balance could be sought through emphasis on more 'Natural Media', or media that promote natural states -a more intuitive, creative and empathetic approach towards the world and each other. In terms of its impact on humans the digital world leads to:

1) Escape from the given reality and as a consequence the abandonment of the body.

2) Transition from biological networking to social, and the development of the social sensorium instead.

3) Escape from the physical time and space continuum. (Miroshnichenko 2016).

The new reality is no longer "given" but it is also not the product of the creative imagination and does not reside in the mind -it is induced, and it is the mind that is now being the representation, which resides within the new "reality" of an artificial world. Through a represented world, we are led, due to media evolution, to an induced one. 


\begin{abstract}
"Video games and social media pave the way inserting a 'representative' of the user in the reality of the game or social interaction." (Miroshnichenko 2016).
\end{abstract}

Augmented reality plays a central role in all this: It is with it that media starts reshaping reality, literally, directly and immediately". We no longer just speak of augmented senses but an augmented reality. Previous media have reshaped and influenced reality only metaphorically. Five sense simulation still uses the physical simulation of nerve endings. But the time is coming for the simulation of nerve "beginnings" not just endings. While the creation of an imaginary world (in the human brain) has always been the end of the arts, literature and the media, they always did this metaphorically. Brands and entertainers now seek to immerse consumers in the experience of a natural-like reality that is fully artificial. The dangers are obvious -we shape our tools, but the tools, in turn, reshape us. The induced reality can capture humans without any use of their imagination, literally, as a surrounding environment/echo chamber.

Literacy gave humankind the ability to function in two worlds, that of the senses (the real) and that of representation (the world of ideas). The predominance of digital media, however, cultivates the tendency to live through representations, with dire consequences, which are already apparent in phenomena such as Hikikomori. From the world of inner vision we might end up living in a schizophrenic society or even an entirely desensitised one. This is what emphasis on natural media can help us prevent.

To summarise, the need for 'Natural Media' is sought as we are living in an increasingly mediated world. Thus, we are becoming dependent on digital systems that create narrow perceptual channels and bottlenecks. For instance, current mobile technology predominantly narrows our focus and makes us oblivious to what is going on around us. We have a vast perceptual bandwidth, which is gradually being shut down and eliminated by the predominance of screen-based interaction.

If the media we consume are fundamentally flawed, then according to the dictum 'the medium is the message' the content we are trying to convey will never quite reach its destination intact (with its full intended impact) but rather will remain a surface representation.

We already spend vast portions of our lives submerged within different types of media but now due to the popularity of increasingly immersive formats such as virtual reality (VR) we intend to immerse ourselves completely within these digital realities. This research urges caution: Before we proceed it is necessary to explore what is fundamentally wrong with the design of the media that we currently use so we can accurately inform the design of future peripheral media/perceptual technologies.

It is a common experience to register activity at the edges of our vision, our peripheral vision (to 'see out of the corner of one's eye') but this concept is not often discussed relative to our other senses. What exactly is peripheral hearing? What about peripheral touch, smell or taste? What would it be like to have access to the periphery of these senses?

In terms of audio, our increasingly predominant choice of media is using lossy MP3 files to deliver our audio experiences where we lose the peripheral experience (full dynamic range of analogue recorded audio), which provides a full spatial experience of being with the recording artists in the studio.

It is becoming increasingly possible to witness a tension between our experience of photographs, films and the actual objects that they represent. There is a growing dissatisfaction with our imaging technologies' inability to capture our subjective experience of reality (even though they capture objective reality quite well - link quote). Linear perspective underpins every imaging technology, but is fundamentally flawed. The eye now adjusts so readily to the effects of linear perspective that it is considered to be an innate process. We are all so familiar with the narrow field-of-view of photographs that we think they are natural. Crucially, the viewer had to become familiar with and learn about perspectival images before they could seem natural. Looking back over the History of Art it appears that linear perspective created an impoverished view of representing experience. This way of imaging the world leads to a sense of remoteness.

\section{FOVOGRAPHY}

The aim of Fovolab's research is to improve the way visual experience is depicted in imaging media by increasing its naturalism. Numerous technical methods for depicting the visual world currently exist. Most of them rely on the optical laws of light rays projected onto a plane (Kingslake 1992). Physical cameras and computer graphics rendering systems are the most common examples. They are designed to accurately capture or computationally simulate the patterns of light emitted or reflected by objects in the world. The images they produce can be thought of as objectively realistic. However, such images do not represent what a human 
experiences when viewing the same objects. The human visual system is not a camera. While there are some similarities between eyes and cameras, much of what we visually perceive is the result of complex cognitive and neurophysiological processing occurring in the visual areas of the brain, for which there is no parallel in current imaging technology (Palmer 1999). This paper briefly outlines our new approach to creating naturalistic media based on the emulation of human visual processes and how this can improve the depiction of the visual world.

\section{NATURAL VISUAL EXPERIENCE}

Humans with healthy vision have two eyes that together cover an angle of view of about 180 degrees horizontally and 130 degrees vertically. (Howard \& Rogers 1995). This visual field is roughly oval in shape, and comprises the contents of both eyes that fuse to form what we perceive as a single image. Eyes, heads and bodies can all move independently from each other with large degrees of freedom, and eyes themselves have several mechanisms for adjusting to visual conditions, including vergence, accommodation, saccadic motion, and pupillary dilation. It has been argued that visual space is non-Euclidian, meaning that there is a non-uniform distribution of object distances, sizes and shapes across the visual field (Hecht et al. 1999). The peripheral areas of the visual field are much less distinct than the central area, which is served by the fovea (Strasburger et al. 2011)

Few of these features of visual perception are incorporated into current imaging technology. Instead, cameras and most common computer graphics rendering systems are designed to capture or computationally simulate the physical properties of light rays that travel through a pinhole and intersect with a plane. Linear perspective, discovered by artists and architects in the fifteenth century, was the first method of formalising this process (Kemp 1990). It held the promise, in theory at least, of accurately representing the threedimensional world on a plane. But its many limitations soon became apparent. It proved useful only for depicting relatively narrow angles of view (normally $<50$ degrees horizontally). It lacks the binocular properties of natural vision. It is static, and relies for its greatest effect on the viewer adopting a motionless, one-eyed viewing point that in practice is almost impossible to obtain. Moreover, linear perspective does not discriminate between the differing visual qualities of the central and peripheral fields. Nor does it accommodate the non-Euclidean structure of visual space (Pepperell \& Hughes 2015)

\section{THE EMERGENCE OF SINGLE-POINT PERSPECTIVE}

The Florentine architect Filippo Brunelleschi is credited with the introduction of a mathematicallydefined single point perspective, although Italian artists from the 1300s onwards were making use of perspectival techniques in their paintings and the concept of the camera obscura was known as early as Ancient China. After Brunelleschi, the artists of the High Renaissance made accurate perspective an essential part of their work, such that it persisted in Western art until the advent of photography enabled artists to explore more abstract visual approaches. The historian and theorist Hubert Damisch characterises perspective's impact as follows:

Perspectiva artificialis does not imitate vision, any more than painting imitates space. It was devised as a means of visual presentation and has meaning only insofar as it participates in the order of the visible, thus appealing to the eye. (Damisch 1994)

However, he also considers Ernst Mach's attempt to reproduce exactly what the eye sees as flawed, because "the painted image is itself given to vision, just like any other object in the visible world, and is thus governed by the rules of vision, with all the consequences it entails." (Damisch 1994). Insofar as Fovography incorporates something of Mach's vision, it must answer this criticism; yet its incorporation of both painterly knowledge and computer processing enables it to approach at least some aspect of a more naturalistic rendering of images. In this, Fovography is recapitulating part of the history of perspective, as related by Brian Winston:

The West's "discovery" or "invention" of perspective may have been a moment as technologically driven as any other. It could have been born, in part, out of knowledge of the camera obscura. Just as Nam June Paik's work (or that of any contemporary artist) is technologically derived today, Brunelleschi's work may have been derived from the technologies of his era (Winston 1987).

\section{THE FOVOGRAPHY METHODOLOGY}

The general approach of Fovography is to model media as closely as possible on actual visual experience. We aim to record what a human sees, not what a camera sees. In practice this means analysing the structure of human visual experience through various methods, including artistic observation and scientific investigation. Having observed the general features of this structure, we then attempt to define it with a series of image composition rules model those rules computationally and finally replicate it techno- 
logically. The resulting images are then tested experimentally to validate the model.

Through this process we have identified a set of key features of natural visual experience that, when digitally synthesised in imaging media, show an improvement across a range of objective measures when compared to images created with conventional perspective methods. These key features include a novel gaze contingent interactive and non-linear projective geometry that reflects more closely the structure of visual space than linear perspective; gaze contingent degradation in the periphery; simulation of physiological diplopia, modification of luminance, contrast and other image variables; and bespoke methods of physical display, projection, and interaction (Burleigh \& Pepperell 2014).

\section{EVIDENCE FOR THE METHODOLOGY}

There is now converging scientific evidence that Fovography can produce an image that is rated as superior to conventional images when measured on a range of criteria. For example, eye tracking studies have shown significantly improved depth perception in Fovographs versus photographs (Baldwin 2016); psychophysical studies have demonstrated the non-Euclidean structure of visual space across the far peripheral field (Baldwin et al. 2016); measures of image comfort, immersion and accuracy using the Spatial Presence Inventory have shown a significant preference for Fovographs over fisheye and linear perspective depictions of wide fields of view (Ruta et al. 2016).

\section{USES OF FOVOGRAPHY IN THE AREA OF MINDFULNESS}

Mindfulness is a technique for focusing attention on what is going on in and around us, thus broadening awareness and countering the 'tunnel vision' associated with stress and anxiety. By representing the periphery of a scene in visual media we might be able to counterbalance 'narrow objective' styles of attention and therefore behaviour.

By including the body within its perspective, Fovography has the potential to bring a wider and more open awareness to sensations in the body as well as the 'felt sense' of the embodied mind increasing both first person perspective and empathy.

Broadening awareness to the breadth of sensory experience in the present moment also releases attachment towards pre-conceived ideas of the self and the conditioned mind. By altering the image properties of visual and perceptually natural media we can illustrate this and therefore show how it's possible to move the mind away from certain states.

Virtual Reality Exposure Therapy (VRET) is showing promise in treating anxiety, phobias, PTSD and other mental health disorders. Studies show a similar effectiveness when Virtual Reality Therapy is compared to other treatments (e.g., CBT).

Content for immersive technologies often presents a limited field of view when compared to the human visual system and can cause nausea and disorientation issues with the confinement and isolation of Virtual Reality. It does not yet consider the importance of social engagement and the act of observation/curiosity towards our perceptual experience.

In VR and most existing immersive technologies, the viewer's body is not included within the media content, which can promote disorientation as well as a sense of separation rather than connection to the viewer.

\section{FOVOGRAPHY FUTURE DEVELOPMENTS}

Fovography began as an artistic method of capturing the full scope of the human visual field in painting and drawing (Pepperell 2012). It has since evolved into a prototype general-purpose computational method of representing visual space more naturalistically. Our current goal is to turn this into a commercially available computer graphics technology for a wide range of possible applications, including medical imaging, entertainment, communications, photography and cinematography, and robot vision. The benefits to users we anticipate will be images that have greater sense of depth, breadth, presence, and immersion, and that offer a more engaging first person perspective than can be obtained with current media technology.

\section{CONCLUSION}

It is important to focus on more than one predominant sense in order to start realigning and potentially redesigning our perception using Natural Media. As a result, current and future work will explore what is the peripheral space of the other senses such as hearing, touch, taste, and smell, and in addition explore artificial senses such as the recently released "exo-sense", 'The North Sense' (https://cyborgnest.net/products/the-north-sense).

The human brain is not a machine, it is not only a processor. It is vital that some of the information it gathers are registered but not consciously processed. The roots of creativity, intuition and 
imagination are to be found in the subconscious. We need to use the periphery of our senses in order to feed the subconscious mind (which does not process directly). For this we need to shift emphasis from the digital to the analogue, to media that is more "natural"" - that is, media that is not entirely automated and mechanised but allows for the human factor to interfere, experiment and ultimately be in control of the experience of the content, instead of the machine.

\section{REFERENCES}

Baldwin, J., Burleigh, A., Pepperell, R., and Ruta, N. (2016) The Perceived Size and Shape of Objects in Peripheral Vision, i-Perception, JulyAugust

Baldwin, J. (2016) Can artistic methods be used to improve the perception of depth in pictures? $\mathrm{PhD}$ Thesis, Cardiff Metropolitan University, UK.

Burleigh, A. and Pepperell, R. (2014) Improvements in and relating to image making. International Patent Treaty Cooperation, WO2014122477 A1.

Damisch, H. (1994) The Origin of Perspective. MIT Press.

Hecht, H., Doorn, A., and Koenderink, J. J. (1999) Compression of visual space in natural scenes and in their photographic counterparts. Perception \& Psychophysics, 61(7), pp. 1269-1286. DOI: 10.3758/BF03206179

Howard, I. P. and Rogers, B. J. (1995) Binocular Vi- sion and Stereopsis. Oxford University Press.

Innis, H. A. (1950) Empire and Communications. A Project Gutenberg Canada Ebook. Clarendon Press, Oxford, p. 7.

http://www.gutenberg.ca/ebooks/innis-empire/innisempire-00-h.html (accessed 1 June 2017).
Kemp, M. (1990) The Science of Art. London: Yale University Press.

Kingslake, R. (1992) Optics in Photography. Bellingham: SPIE Press.

McLuhan, M. and Fiore, Q. (1967) The Medium Is the Massage: An Inventory of Effects. New York: Random House, p. 48.

Miroshnichenko, A. (2016) Extrapolating on McLuhan: How Media Environments of the Given, the Represented, and the Induced Shape and Reshape our Sensorium. Philosophies, 1.3, pp. 170-189.

Palmer, S. (1999) Vision Science. Cambridge, Mass.: Bradford.

Pepperell, R. (2012) The Perception of Art and the Science of Perception. In Human Vision and Electronic Imaging XVII, B. E. Rogowitz, T. N. Pappas, \& H. de Ridder (eds.), Proc. of SPIE-IS\&T, Elec. Im., Vol. 8291, 829113.

Pepperell, R. and Hughes, L. (2015) As Seen: Modern British Painting and Visual Experience. Tate Papers, Spring.

Ruta, N., Burleigh, A., Vigars, R., Barratt, E., and Pepperell, R. (2016) Evaluating an artistic method for depicting human visual space. Applied Vision Association meeting, London, 19 December.

Shirky, C. (2010) Cognitive Surplus: Creativity and Generosity in a Connected Age. New York: The Penguin Press, p. 54.

Strasburger, H., Rentschler, I., and Jüttner, M. (2011) Peripheral Vision and Pattern Recognition: A review. Journal of Vision, 11(5), pp. 1-82.

Winston, B. (1987) A Mirror for Brunelleschi. Daedalus, 116(3), pp. 187-201, Futures, Summer. 